\title{
Coordinated optical and EISCAT observations of black aurora
}

\author{
E. M. Blixt \\ Department of Physics, University of Tromsø, Tromsø, Norway \\ M. J. Kosch \\ Department of Communications System, Lancaster University, Lancaster, UK
}

Received 9 December 2003; revised 3 March 2004; accepted 8 March 2004; published 31 March 2004.

[1] An image intensified video camera was used in conjunction with the EISCAT radar in Tromsø, Norway, for detailed studies of non-sheared black aurora on March 5 , 2002. While it is known that a downward field-aligned current will decrease the ionospheric plasma density, any measurable effect will depend on the noise-level in the density measurements and the background density. The results show that the plasma density is not reduced in the regions of black aurora, which shows that if a downward current existed within the black aurora, it must be less than $\sim 0.6 \mu \mathrm{A} / \mathrm{m}^{2}$. Combining this result with recent findings that small scale currents are generally strong, that the downward currents are associated with intense diverging electric fields, and that the observed black arcs show no signs of shear motion, it is concluded that the non-sheared black aurora is not associated with any significant downward field-aligned current. INDEX TERMS: 2467 Ionosphere: Plasma temperature and density; 2409 Ionosphere: Current systems (2708); 2407 Ionosphere: Auroral ionosphere (2704); 2704 Magnetospheric Physics: Auroral phenomena (2407). Citation: Blixt, E. M., and M. J. Kosch (2004), Coordinated optical and EISCAT observations of black aurora, Geophys. Res. Lett., 31, L06813, doi:10.1029/2003GL019244.

\section{Introduction}

[2] Black aurora is recognized as a spatially well defined region within uniform diffuse aurora that lacks optical emissions [Royrvik, 1976; Davis, 1978]. These black regions can be broadly categorised into two groups; those associated with shear motions, which often evolve into black vortex streets [Kimball and Hallinan, 1998b], and black patches which are not associated with shear [Kimball and Hallinan, 1998a].

[3] Black aurora exhibit a variety of forms, from curls and vortex streets (with a sense of rotation opposite to that of auroral curls [Hallinan and Davis, 1970]), to elliptical and stretched patches (black arcs) [Trondsen and Cogger, 1997; Marklund et al., 1997; Kimball and Hallinan, 1998a, 1998b]. Mapped to an altitude of $100 \mathrm{~km}$, black patches are typically around $5 \times 1 \mathrm{~km}$ in extent, are common in the late recovery phase of an auroral substorm, and move predominately in the eastward direction with a speed of 1 to $2 \mathrm{~km} / \mathrm{s}$.

[4] Black vortex structures are understood to be due to downward field-aligned currents (FACs) within regions of narrow diverging electric fields [Royrvik, 1976; Marklund et al., 1997], that is, similar, but opposite, to auroral curls.

Copyright 2004 by the American Geophysical Union. 0094-8276/04/2003GL019244\$05.00
However, our understanding of non-sheared black aurora is less well established. To date no simultaneous optical and in-situ radar observations of black aurora exists, and the generation mechanism for non-sheared black aurora is still under debate. Recent observations by Peticolas et al. [2002] indicate that black patches are found within narrow regions where the loss cone is partially empty of energies larger than $2 \mathrm{keV}$, and they suggest that where scattering into the loss cone is locally hindered, black aurora occurs. This means that black aurora are not associated with downward FAC's but merely the lack of precipitating electrons.

[5] As explained in section 4, it is likely that a downward current within the black aurora would lower the ionospheric density. We present observations of black patches from a coordinated optical-radar experiment during the evening of 5 March 2002 from EISCAT, Tromsø, where we searched for any such density depletions within black aurora using incoherent scatter radar measurements of plasma density combined with an auroral imager to determine the position of the black patches. These are the first reported observations of black aurora using incoherent backscatter radar.

\section{Instrumentation}

[6] EISCAT (the European Incoherent Scatter radar) in Tromsø $\left(69.58^{\circ} \mathrm{N}, 19.22^{\circ} \mathrm{E}\right)$ was used for direct observations of ionospheric electron density. EISCAT was pointing along the local magnetic field line, and ran the tau 3 programme, which yields a time resolution of $5 \mathrm{~s}$, and an unambiguous range resolution of $5.4 \mathrm{~km}$. The radar beam has a full opening angle of about $0.6^{\circ}$, which corresponds to about $1 \mathrm{~km}$ at the altitude of typical auroras $(105 \mathrm{~km})$.

[7] For visual observations of the black patches the Optical digital imager (Odin) of University of Tromsø was used. The video signal is digitized in real time and synchronized through GPS satellites to achieve exact timing. A F0.95 $50 \mathrm{~mm}$ lens was used, which yields a $14.3 \times 10.9^{\circ}$ field of view.

\section{Observations}

[8] The Odin imager and the EISCAT radar was running in parallel on the evening of 5 March 2002, when several black arcs where observed between 2000 and 2100 UT. Geomagnetic activity was high $(\mathrm{Kp}=5)$, and the observations took place after an auroral breakup which terminated at around 1930 UT.

[9] Between 2004:11 and 2004:30 (event A), and 2013:53 and 2014:46 UT (event B) black arcs were seen to enter the radar beam. The black arc in event $\mathrm{A}$ was about 


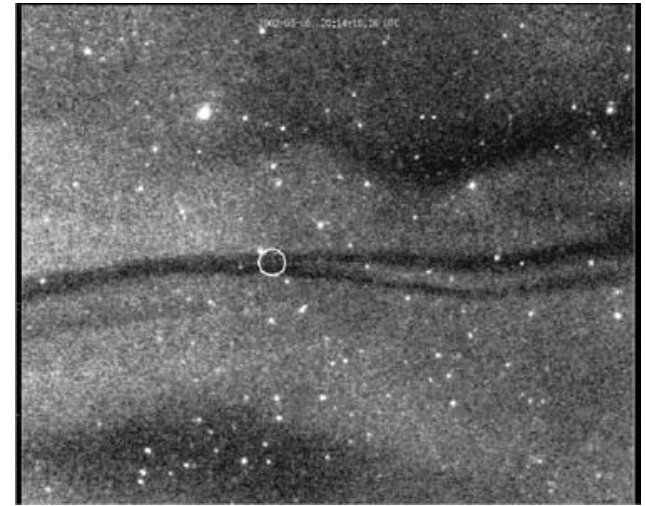

Figure 1. An image of a black arc passing through the radar beam (white circle) at 2014:16 UT.

$1.5 \mathrm{~km}$ wide, and moved southward at $\sim 0.1 \mathrm{~km} / \mathrm{s}$. The black arc in event B moved slowly northward $(\sim 0.05 \mathrm{~km} / \mathrm{s})$, and deformed slightly from being $\sim 2 \mathrm{~km}$ wide to $\sim 1 \mathrm{~km}$ wide during its passage through the radar beam. The black arc in event A never filled the radar beam completely, while the arc in event B occupies the whole radar beam for at least $10-15$ s (see Figure 1).

[10] By plotting the total light intensity within the radar beam (so called in-beam luminosity) as function of time we see in Figures 2 and 3 how the presence of the black arcs lowers the observed intensity. Note that the auroral imager is not calibrated, so the background level is not known in the luminosity plots.

[11] As can be seen from Figure 2, the black arc in event A reaches the radar beam at about 2004:10 UT. The black arc passed rather quickly through the radar beam and the inbeam luminosity was not greatly affected. In event B the thin black arc moved through the radar beam around 2013:55 UT. A clear drop in in-beam luminosity is seen in Figure 3 for nearly $60 \mathrm{~s}$. Both black arcs were observed for about 2 minutes. During that time no signs of shear flow in the surrounding diffuse aurora were observed.

[12] Figure 4 shows the $30 \mathrm{~s}$ integrated electron density measured by EISCAT. The noise level is clearly visible although the integration does increase the signal-to-noise ratio (SNR) significantly. The median SNR is about 20, with a standard deviation of about 15 .

\section{Discussion}

[13] Auroral arcs are associated with a matched pair of FACs, the upward current centered above the arc while the

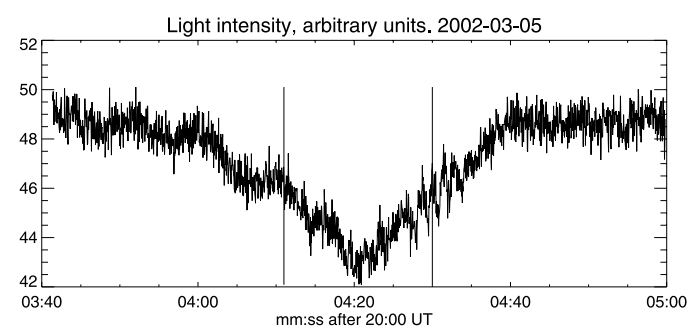

Figure 2. Total light intensity observed by the imager within the radar beam. The two vertical lines shows when a black arc was seen to enter the radar beam in event $\mathrm{A}$.

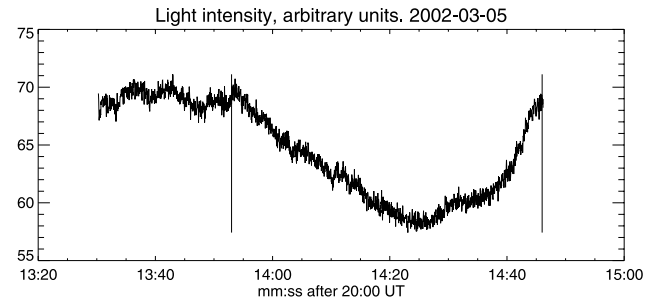

Figure 3. Same as Figure 2, but for event B.

downward current is found either poleward or equatorward of it depending on the direction of the background convection electric field. In the region of the downward current it is common to observe enhanced electric fields [Marklund et al., 1981; Brüning and Goertz, 1986] and low ionospheric plasma density, which is understood to be an effect of current continuity through the arc boundary [Doe et al., 1995; Blixt and Brekke, 1996].

[14] The same effect should be present in black aurora if they are associated with downward currents. We expect a low plasma density within the black region because the downward current is carried by upward moving thermal electrons of ionospheric origin, while the closing horizontal Pedersen current is carried by ions, leaving behind a depleted region.

[15] To study the loss process due to a downward current, we start with the ion continuity equation, but neglect recombination which only increases the loss. We adopt a simplified "box" like ionosphere of height $h$, which is homogeneous in the direction parallel to the black arc (like that shown in Figure 1).

[16] To derive the relationship between the ionospheric plasma density and the FAC, one further assumes that the Pedersen current is mainly carried by ions in the collisionally dominated ionosphere, and that the downward fieldaligned current is mainly carried by upward moving cold ionospheric electrons. The detailed derivation was treated by Blixt and Brekke [1996] and is not repeated here. It yields:

$$
\frac{\partial}{\partial t} n \approx \frac{j_{\|}}{h e}
$$

where $n$ is the average plasma density in the region of the ionosphere carrying the closure current ( $\approx$ E-region), $e$ is the

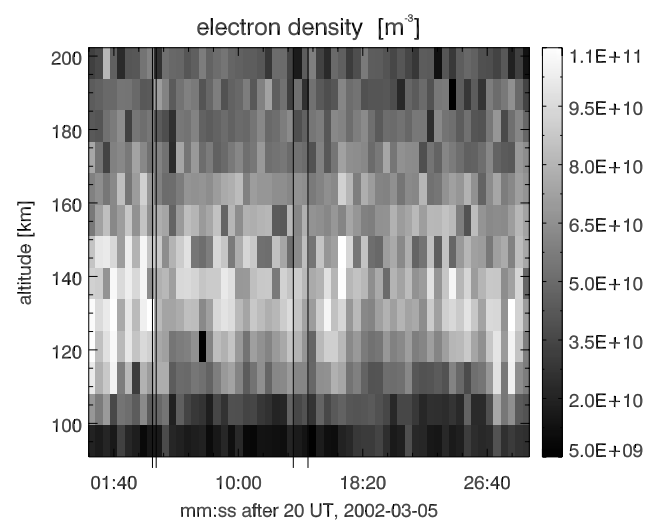

Figure 4. Electron density measured by EISCAT. The four vertical lines outline events $\mathrm{A}$ and $\mathrm{B}$. 


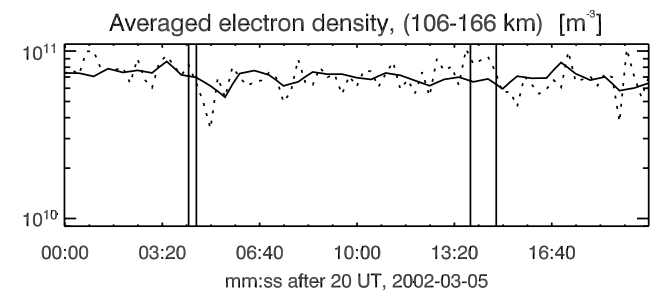

Figure 5. Average electron density variation with time. The solid line shows the $30 \mathrm{~s}$ integrated data while the dotted lined shows the $15 \mathrm{~s}$ integrated data. The average is taken from 106 to $166 \mathrm{~km}$ altitude and the vertical lines show events $\mathrm{A}$ and $\mathrm{B}$.

electron charge, and $j_{\|}$is the downward FAC. By integrating equation (1) from when the depletive mechanism becomes active $\left(t_{0}\right)$ to $t_{1}=t_{0}+\tau$ (assuming the FAC to be constant) we get

$$
j_{\|} \approx \frac{n_{0} h e}{\tau}\left(\frac{n_{1}-n_{0}}{n_{0}}\right) .
$$

Thus, equation (2) estimates how large the current density must be to yield a given density depletion $\left(\left(n_{1}-n_{0}\right) / n_{0}\right)$. Equation (2) cannot be used in the upward current region, as precipitation causes considerable additional ionization.

[17] For the effect of the depletive mechanism to be seen in noisy electron density data, the depletion must be greater than the radar noise level given by the SNR, thus $\left|\left(n_{1}-n_{0}\right)\right|$ $n_{0} \mid>1 / S N R$. By using this in equation (2) we get

$$
\left|j_{\|}\right|>\frac{n_{0} h e}{\tau}\left(\frac{1}{S N R}\right) .
$$

To estimate the time the depletive mechanism is active $(\tau)$ within the diffuse aurora we must know if the downward current of the black aurora moves with the ionospheric plasma $(E \times B$ velocity corresponds to the motion of the aurora), or if the current system moves relative to the ionospheric plasma. In the first case, $\tau$ corresponds to the effective lifetime of the black aurora, while in the latter case $\tau$ can be approximated with the halfwidth of the black arc divided by the relative motion of the black segment. Any relative motion requires a potential drop along the magnetic field, and the lack of any optical signatures of velocity shears indicates that the potential drop in this case (if any) is very small. The diffuse aurorae, which contain the black patches, are large scale features. It is known that large scale electric fields map directly along the magnetic field [Lyons, 1980], while it is only within small scale auroral arcs that we can expect a differential motion between the aurora and the ionospheric plasma. We therefore argue that the effective time of the mechanism is equal to the lifetime of the black patch. Black patches and arcs have much longer lifetimes than black vortex streets. Kimball and Hallinan [1998a] noticed that black patches generally survive the time it takes to pass through the imager's field of view (5 to $10 \mathrm{~s}$ ) but also tracked individual patches for up to a minute in all-sky imager data. As noted by Davis [1978] black patches can not be observed more than $30^{\circ}$ off-zenith, which limits their observing duration. In contrast, the black aurora observed here move slowly and last up to $120 \mathrm{~s}$, so it is realistic to assume a lifetime of about 30 to $90 \mathrm{~s}$.

[18] We expect the depletion to be most effective in the E-region where the conductivity peaks (or more exactly, the region where the electron density is enhanced, which is where perpendicular currents will close in the ionosphere). As seen from Figure 4 this approximately corresponds to the 106 to $166 \mathrm{~km}$ altitude region $(h=60 \mathrm{~km})$. The height averaged electron density of this altitude region is shown in Figure 5 for $30 \mathrm{~s}$ integrated data (solid line) and $15 \mathrm{~s}$ integrated data (dotted line). The electron density is around $n \approx 8 \cdot 10^{10} \mathrm{~m}^{-3}$ and the additional height averaging over 7 radar range gates further improves the electron density SNR, increasing it from 20 to around 50 in the $30 \mathrm{~s}$ integrated data.

[19] By inserting the above values into equation (3) we obtain the results shown in Figure 6. It is evident that for a black auroral life time of $60 \mathrm{~s}$ (which is likely), and for $\mathrm{SNR} \approx 50$, the current needs to be stronger than $0.3 \mu \mathrm{A} / \mathrm{m}^{2}$ for it to yield a density depletion deep enough to be seen through the noise in the radar data. We have chosen to use the SNR level from the $30 \mathrm{~s}$ integrated EISCAT data because, as discussed in more detail below, the time resolution of that experiment is sufficient in resolving the black auroral passage in event B.

[20] Marklund et al. [2001] observed currents in the range of $0.1-0.2 \mu \mathrm{A} / \mathrm{m}^{2}$ in the downward FAC adjacent to auroral arcs with the Cluster satellites. This corresponds to $10-20 \mu \mathrm{A} / \mathrm{m}^{2}$ at ionospheric altitudes, but at a horizontal scale an order of magnitude larger than the black aurora. Kosch et al. [2000] observed downward current densities of $\sim 1 \mu \mathrm{A} / \mathrm{m}^{2}$ adjacent to an auroral arc. While a typical strength for upward currents associated with individual auroral arcs is around $1-10$ 's $\mu \mathrm{A} / \mathrm{m}^{2}$, recent studies indicate that the current strength is roughly inversely proportional to scale size [Stasiewicz and Potemra, 1998], and currents up to several 100's (even 1000) $\mu \mathrm{A} / \mathrm{m}^{2}$ have routinely been observed [Stasiewicz and Potemra, 1998; Neubert and Christiansen, 2003] on sub-kilometer scales onboard lowaltitude satellites. Marklund and Karlsson [2001] argue that the current can be as strong as $30 \mu \mathrm{A} / \mathrm{m}^{2}$ within a black aurora. Our observations and estimates of the FAC strength makes it likely that if black aurora were associated with a FAC, the depletive effect would be seen in our data.

[21] As can be seen in Figure 5 there are several occasions when the plasma density exhibits a minor decrease. The largest decrease occurs right after event A (about 1 minute later), and there is also a smaller one after event

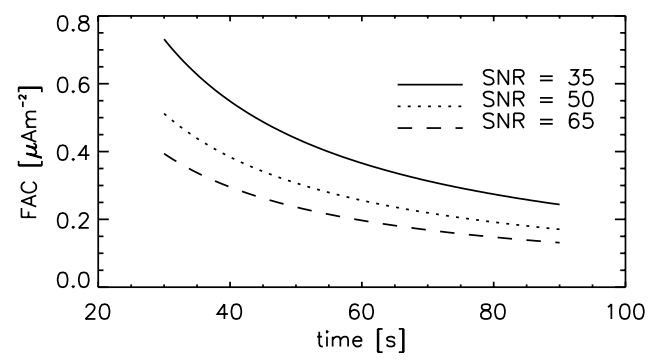

Figure 6. The time a downward current density, for a given SNR, can be active before it yields a density depletion large enough to be detected above the radar noise. 
$\mathrm{B}$, but it is clear that the direct passage of the black arcs does not alter the electron density significantly. One possible cause for this could be that the black arc only partially fills the radar beam, so that the whole volume covered by the radar beam is not depleted by the same amount. We can take this into account by assuming the worst case scenario. The passage of the black arc in event B takes nearly two full radar integration periods (60 s). As the black arc has about the same width as the radar beam, we can assume that it only fills half the radar beam for two consecutive radar integration periods.

[22] Another possibility is that the black arc is associated with a very weak current, or no current at all. Event A is probably too short lived (the black arc passes through the radar beam in less than $30 \mathrm{~s}$ ) to give an observable effect. However, for event B, if we take into account the factor of two in the worst case scenario, we find that the current must be less than $0.6 \mu \mathrm{A} / \mathrm{m}^{2}$. We note that this is an overestimate, since the assumed black aurora lifetime of $60 \mathrm{~s}$ is relatively short compared to the observed lifetime up to $120 \mathrm{~s}$. Since currents at small spatial scales, like that of the black aurora, are found to be much stronger than large scale currents, it is very likely that the black patches presented in this paper were not associated with any significant downward FAC.

[23] Marklund et al. [2001] showed that the downward current region is characterized by intense diverging electric field structures at the typical altitudes of the Freja satellite, and Marklund et al. [1997] associated these downward currents with black aurora. It is well established that the sheared motions (and curls) found in auroral arcs are due to converging electric fields. The lack of any shear motion in the diffuse aurora surrounding the black aurora presented here is because they are not associated with any intense diverging electric field. We interpret this as another indicator that the black arcs are not associated with any downward current.

\section{Conclusion}

[24] Results from the first coordinated optical and incoherent scatter radar campaign for observations of black aurora are presented.

[25] We show that the presence of a downward fieldaligned current would give rise to a decrease in the ionospheric electron density, and if the current strength is over a certain threshold (depending on the signal-to-noise ratio in the electron density data) the decrease would be measurable by the EISCAT radar. Black aurora are often claimed to be associated with a downward FAC, and would in this case, by the argument above, be associated with a plasma density decrease.

[26] Observations of two black arcs that drift through the radar beam are discussed. The first passage is short lived, while the second passage lasts for about a minute. No evidence of an electron density decrease during the black arc passages was seen in the EISCAT measurements.
[27] We show that if a downward FAC exists within these black aurora, it must be less than $\sim 0.6 \mu \mathrm{A} / \mathrm{m}^{2}$. Based on the fact that small scale currents are generally stronger than large scale currents $\left(10-100\right.$ 's of $\mu \mathrm{A} / \mathrm{m}^{2}$ as compared to $<1 \mu \mathrm{A} / \mathrm{m}^{2}$ for large scale currents), and that the black arcs are not sheared and thus not associated with intense diverging electric fields, we conclude that the black arcs presented here are not associated with any significant downward fieldaligned current.

[28] Acknowledgments. We gratefully acknowledge Andrew Senior for assisting with the analysis of the radar data. The EISCAT Scientific Association is supported by CNRS of France, the Max-Planck-Gesellschaft of Germany, PPARC of the United Kingdom, NFR of Norway, NFR of Sweden, Suomen Akatemia of Finland and NIPR of Japan.

\section{References}

Blixt, E., and A. Brekke (1996), A model of currents and electric fields in a discrete auroral arc, Geophys. Res. Lett., 23(18), 2553-2556.

Brüning, K., and C. Goertz (1986), Dynamics of a discrete auroral arc, J. Geophys. Res., 91, 7057-7064.

Davis, T. (1978), Observed characteristics of auroral forms, Space Sci. Rev., $22,77-113$

Doe, R., J. Vickrey, and M. Mendillo (1995), Electrodynamic model for the formation of auroral ionospheric cavities, J. Geophys. Res., 100, $9683-$ 9696.

Hallinan, T., and T. Davis (1970), Small-scale auroral arc distortions, Planet. Space Sci., 18, 1735-1744.

Kimball, J., and T. Hallinan (1998a), Observations of black auroral patches and of their relationship to other types of aurora, J. Geophys. Res., 103, $14,671-14,682$.

Kimball, J., and T. Hallinan (1998b), A morphological study of black vortex streets, J. Geophys. Res., 103, 14,683-14,695.

Kosch, M., O. Amm, and M. Scourfield (2000), A plasma vortex revisited: The importance of including conductivity measurements, J. Geophys. Res., 105, 24,889-24,898.

Lyons, L. (1980), Generation of large-scale regions of auroral currents, electric potentials, and precipitation by the divergence of the convection electric field, J. Geophys. Res., 85, 17-24.

Marklund, G., and T. Karlsson (2001), Characteristics of the auroral particle acceleration in the upward and downward current regions, Phys. Chem. Earth Part C., 26, 81-96.

Marklund, G., L. Block, and P. Lindquist (1981), Rocket measurements of electric fields, electron density and temperature during different phases of auroral substorms, Planet. Space Sci., 29, 249-259.

Marklund, G., T. Karlsson, and J. Clemmons (1997), On low-altitude particle acceleration and intense electric fields and their relationship to black aurora, J. Geophys. Res., 102, 17,509-17,522.

Marklund, G., et al. (2001), Temporal evolution of the electric field accelerating electrons away from the auroral ionosphere, Nature, 14, 724727

Neubert, T., and F. Christiansen (2003), Small-scale field-aligned currents at the top-side ionosphere, Geophys. Res. Lett., 30(19), 2010, doi:10.1029/2003GL017808.

Peticolas, L. M., T. J. Hallinan, H. C. Stenbaek-Nielsen, J. W. Bonnell, and C. W. Carlson (2002), A study of black aurora from aircraft-based optical observations and plasma measurements on FAST, J. Geophys. Res., 107(A8), 1217, doi:10.1029/2001JA900157.

Royrvik, O. (1976), Pulsating aurora: Local and global morphology, Ph.D. thesis, Univ. of Alaska, Fairbanks.

Stasiewicz, K., and T. Potemra (1998), Multiscale current structures observed by Freja, J. Geophys. Res., 103, 4315-4325.

Trondsen, T., and L. Cogger (1997), High-resolution television observations of black aurora, J. Geophys. Res., 102, 363-378.

E. M. Blixt, Auroral Observatory, University of Tromsø, N-9037 Tromsø, Norway. (marten.blixt@phys.uit.no)

M. J. Kosch, Department of Communications System, Lancaster University, Lancaster, UK. 